\title{
Delayed Onset of Seizures and Toxicity Associated with Recreational Use of Bromo-dragonFLY
}

\author{
David M. Wood, MD, MRCP(UK)a, Jemma J. Looker, BSc, MB BSb, Loua Shaikh, BSc, MB ChBb, \\ Jenny Button, DipFMSc, Malgorzata Puchnarewicz, MPharmc, Susannah Davies, BSc, MScc, \\ Satnam Lidder, MD ${ }^{a}$, John Ramseyd, David W. Holt, PhD, DSc c, Paul I. Dargan, FRCPEa
}

aClinical Toxicology, Guy's and St. Thomas' NHS Foundation Trust, London, UK

bIntensive Care Medicine and Anaesthetics, Frimley Park NHS Foundation Trust, Frimley, UK

cAnalytical Unit, St George's, University of London, London, UK

dTICTAC Communications Ltd, St. George's, University of London, London, UK

\begin{abstract}
Introduction: Many countries have specific legislation, such as the Controlled Substances Act (1970) in the United States and the Misuse of Drugs Act (1971) in the United Kingdom to control recreational drugs. There is a growing market and supply of "novel" recreational drugs, which include the misuse of pharmaceutical compounds and research chemicals. These are often not covered under current legislation, despite the fact that they often have both similar chemical structures and/or clinical effects to controlled recreational drugs.

Case Report: A male patient presented to an emergency department with delayed onset of severe agitation, hallucinations, and tonic-clonic seizures following the use of Bromo-dragonFLY and an unknown white powder. He settled following IV benzodiazepines and supportive care, and was discharged with no evidence of long-term sequelae. Analysis of the white powder by gas chromatography/mass spectrometry (GC/MS), ultraviolet/visible spectrophotometry (UV/VIS) and thin layer chromatography (TLC) showed the presence of Bromo-dragonFLY (1-(8-bromobenzo[1,2-b;4,5-b']difuran-4-yl)-2-aminopropane); serum analysis by GC/MS and liquid chromatography with tandem mass spectrometry (LC/MS/MS) confirmed that a combination of Bromo-dragonFLY (0.95 ng/mL), ketamine $(20 \mathrm{ng} / \mathrm{mL})$ and cannabis had been used by the patient. No other recreational drugs were detected in an extensive toxicological screen of serum and urine samples.

Discussion: This is the first confirmed case to be reported of toxicity with delayed onset of severe agitation, hallucinations and tonic-clonic seizures associated with recreational use of Bromo-dragonFLY (1-(8-bromobenzo[1,2-b;4,5-b']difuran-4-yl)-2-aminopropane) in combination with ketamine and cannabis. In our view, this case provides further support for the need for a systematic approach to toxicological screening of patients with recreational drug toxicity, to identify emerging drugs and provide evidence for legislative authorities to assist in revising the legal status of emerging recreational drugs.
\end{abstract}

Keywords: Bromo-dragonFLY, recreational drugs, toxicological screening, ketamine

Notes: This case report has not previously been presented.

D.W. and P.D. have acted as scientific advisors to the UK Advisory Council on the Misuse of Drugs (ACMD) and the European Monitoring Centre for Drugs and Drug Addiction (EMCDDA).

There was no outside funding of any kind used for this study.

The authors have no potential financial conflicts of interest to report.

Corresponding Author: Dr. David Wood, Consultant Physician and Clinical Toxicologist, Medical Toxicology Office, 2nd Floor, Bermondsey Wing, Guy's Hospital, Great Maze Pond, London, SE1 9 RT, UK. Email: David.Wood@gstt.nhs.uk 


\section{INTRODUCTION}

Recreational drugs are controlled under relevant drug legislation such as the Controlled Substances Act (1970) in the United States and the Misuse of Drugs Act (1971) in the United Kingdom. In spite of this legislation, there have been many recent reports of toxicity associated with emerging "novel" recreational drugs or pharmaceutical agents misused as recreational drugs [1-6]. Often the legislation concerning these novel recreational drugs is inconsistent, with control only occurring in a limited number of countries [4].

Legislative authorities are often unaware of what products and chemicals are being marketed and/or sold as recreational drugs. In addition, there is often limited or no clinical information on the toxicity of these products and compounds and often the information is available only through uncontrolled self-reports by recreational drug users on user websites or blogs. We report here a confirmed case of recreational use of Bromo-dragonFLY (1-(8-bromobenzo[1,2-b;4,5-b']difuran-4-yl)-2-aminopropane), a research chemical currently only formally controlled in Sweden and Denmark, which was associated with delayed onset of severe agitation, hallucinations, and tonic-clonic seizures.

\section{CASE REPORT}

An 18-year-old male with a past medical history of mild asthma controlled on inhaled salbutamol and Flixotide (fluticasone propionate, Allen and Hamburys Ltd), presented following ingestion of some Bromo-dragonFLY and insufflation of an unknown white powder; the exact amounts taken were not known. The BromodragonFLY and white powder were taken at 11:00 PM and initially he reported developing hallucinations. Approximately 8 hours following ingestion, he became increasingly agitated with worsening hallucinations, and his friends called an ambulance as they were unable to calm him down.

On arrival of the ambulance crew, he had 2 witnessed selfterminating generalized seizures with associated apnea. Following transfer to the emergency department (ED), he was unconscious with a Glasgow Coma Score of $3 / 15$, a heart rate of $124 \mathrm{bpm}$, and blood pressure of $182 / 94$. He was apyrexial $\left(36.2^{\circ} \mathrm{C}\right)$ and had oxygen saturations of $97 \%$ on room air, with a respiratory rate of 26 breaths/minute. His pupils were dilated $(6 \mathrm{~mm})$ bilaterally and poorly reactive to light. Shortly after arrival, he had a further self-terminating generalized seizure lasting approximately 1 minute, followed by another generalized seizure that was treated successfully with a single dose of $4 \mathrm{mg}$ IV lorazepam.

His admission electrocardiogram (ECG) showed a sinus tachycardia, and initial laboratory biochemical tests were: potassium, $5.9 \mathrm{mmol} / \mathrm{L}(5.9 \mathrm{mEq} / \mathrm{L})$; creatinine, $140 \mu \mathrm{mol} / \mathrm{L}(1.58 \mathrm{mg} / \mathrm{dL})$; and glucose, $12.0 \mathrm{mmol} / \mathrm{L}(218.2 \mathrm{mg} / \mathrm{dL})$. An initial arterial blood gas following his seizures showed evidence of significant combined metabolic and respiratory acidosis: $\mathrm{pH}$ 6.88, PaCO2, 13.8 kPa (103.8 mmHg); PaO2, 39.6 kPa (297.7 mmHg); bicarbonate, $18.5 \mathrm{mmol} / \mathrm{L}$ (18.5 mEq/L), base excess, -13.1; lactate, $8.5 \mathrm{mmol} / \mathrm{L}$
(76.6 $\mathrm{mg} / \mathrm{dL}$ ) on $15 \mathrm{~L}$ of oxygen via a reservoir bag facemask. Therefore, he was intubated and ventilated and a CT brain scan performed, which showed no evidence of intracranial pathology; in particular, there was no evidence of intracranial hemorrhage.

Further advice on his management was sought from Guy's and St Thomas' Poisons Unit, who suggested that in view of the 5HT2A activity of Bromo-dragonFLY he should be monitored for signs of serotonin toxicity. The advice of the Poisons Unit was that should he develop any signs of serotonin toxicity, he should be treated initially with IV benzodiazepines and fluids. If he was not settling on this management, he should then be treated with oral cyproheptadine (12 mg followed by 2 mg every 2 hours until asymptomatic). He was admitted to the intensive care unit for ongoing management. His respiratory and metabolic acidosis settled: pH, 7.36; PaCO2, $5.11 \mathrm{kPa}(38.4 \mathrm{mmHg}) ; \mathrm{PaO} 2,20.5 \mathrm{kPa}$ (154.1 mmHg); bicarbonate, $21.1 \mathrm{mmol} / \mathrm{L}(21.1 \mathrm{mEq} / \mathrm{L})$; base excess, -3.4 on $4 \mathrm{~L}$ inspired oxygen the day after admission. He did not develop signs of serotonin toxicity. He developed signs of decreased air entry at his right lung base, consolidation on his chest radiograph, and thick mucous secretions through his endotracheal tube and on bronchoscopy, consistent with probable aspiration pneumonia, for which he was treated with intravenous coamoxiclav, gentamicin, and metronidazole. He was extubated within 24 hours of admission, and did not require any additional ventilatory support. He was discharged home 4 days after admission, with advice on recreational drugs and their use.

\section{Toxicological Screening}

A sample of the white powder was sent for toxicological analysis by the Analytical Unit, St George's, University of London. Analysis by gas chromatography/mass spectrometry (GC/MS), ultraviolet/visible spectrophotometry (UV/VIS) and thin layer chromatography (TLC) confirmed the presence of Bromo-dragonFLY. Informed consent was obtained from the patient for toxicological analysis of serum and urine samples collected on admission. Routine toxicological analysis of the serum and urine specimens using GC/MS and liquid chromatography with tandem mass spectrometry (LC/MS/MS) identified the presence of Bromo-dragonFLY (0.95 ng/mL) and ketamine and metabolites (20 ng/mL). Cannabinoids were detected in both serum and urine. Lidocaine, lorazepam, midazolam, and thiopentone were detected as a result of treatment given in the ED. No other drugs or alcohol were detected using a broad toxicology screen of both the serum and urine samples; in particular, there were no drugs (recreational or pharmaceutical) that are associated with the development of the seizures detected.

\section{DISCUSSION}

We have described here the case of an individual who developed severe agitation, hallucinations, and tonic-clonic seizures approximately 8 hours after use of Bromo-dragonFLY. Subsequent toxicological analysis demonstrated that he had, in fact, ingested a combination of Bromo-dragonFLY, ketamine, and cannabis. 
Bromo-dragonFLY is a synthetic psychedelic drug, which has no known therapeutic use in humans, and is currently only available as a "research chemical" from chemical suppliers in China. Currently, there are no known legitimate industrial uses for Bromo-dragonFLY. It is structurally related to phenethylamine, although it is classified within a new class of benzodifurans. There are a number of compounds with a similar chemical structure to Bromo-dragonFLY, differing by substitution of the bromide atom with other entities. The underlying structure for all of these compounds is 2 dihydrofuran rings that are found symmetrically on opposite sides of the central benzene ring, and are said to resemble a dragonfly, hence the generic name. The chemical structure of Bromo-dragonFLY is shown in comparison with serotonin in Figure 1. These compounds have been developed as potent research tools for investigation of the serotonin receptor family, in particular the 5-HT2 subfamily of receptors [7-10]. These agents were thought to have a potential role in the development of novel antidepressant drugs that stimulate release of serotonin, differing from conventional antidepressants that inhibit the reuptake of serotonin.

There are reports that DragonFLY derivatives have been sold on blotters and in solution, thus there is the potential that individuals could believe that they are instead ingesting LSD $[11,12]$. The potency of Bromo-dragonfLY in humans is not clearly understood, although it is likely to be pharmacologically active at very low concentrations. There are several user reports published on websites that describe a longer duration of action than other compounds such as LSD, with a usual duration of action of 12-24 hours, although this can be much longer (up to 72 hours) in some users $[11,12]$. These user website reports also highlight concerns by users about the potential for severe adverse effects including nausea, headache, fever, and a feeling of having a "messed up mind" when this type of compound is used recreationally by inexperienced users. Additionally, users report that the onset of maximal effects following ingestion of BromodragonFLY may not occur for up to 6 hours postingestion [11]. This could explain the delayed onset of severe symptoms seen in our patient, consistent with the previous user reports of delayed onset of action.

Our patient subsequently self-reported ingestion of ketamine in addition to Bromo-dragonFLY, and ketamine was detected on toxicological screening. However, we do not feel that this patient's significant symptoms of agitation and subsequent seizures are likely to have been due predominantly to ketamine, as they occurred over 8 hours following ingestion and this would not be typical of ketamine toxicity [13]. Additionally, symptoms following nasal insufflation typically tend to last only 45-90 minutes. Furthermore, the ketamine concentrations were only $20 \mathrm{ng} / \mathrm{mL}$, which is much lower than that seen in therapeutic use of ketamine and significantly lower than would be expected in patients with toxicity associated with recreational use of ketamine. In a volunteer study investigating the psychological effects of ketamine, the mean ketamine concentration achieved was $209.6 \mathrm{ng} / \mathrm{mL}$

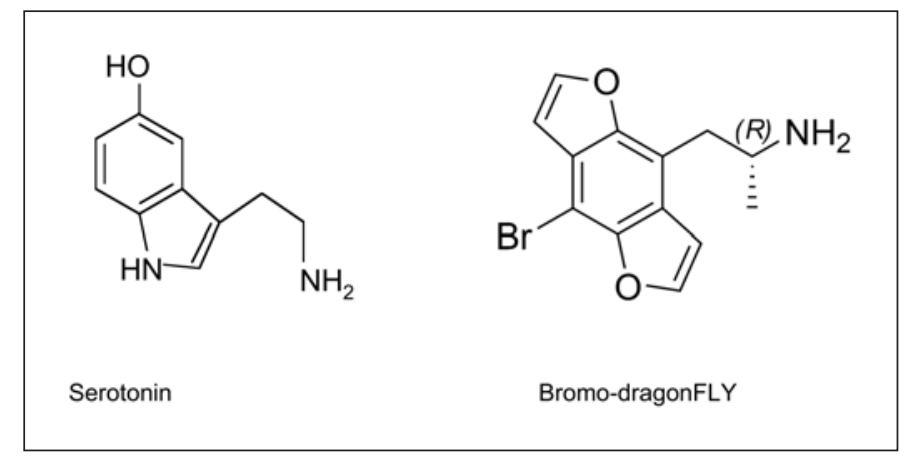

Figure 1: Chemical structures of serotonin and Bromo-dragonFLY.

(range 130.7-303.3 ng/mL), 10 times the concentration in our patient [14]. None of the volunteers experienced agitation, hallucinations, or significant drowsiness at these significantly higher concentrations. In conclusion, we feel that it is likely that the agitation and convulsions in our patient were due to BromoDragonFLY. We cannot exclude the possibility of a drug-drug interaction between ketamine and Bromo-dragonFLY contributing to the clinical presentation in our patient, however.

There are 4 previous cases of toxicity associated with recreational use of Bromo-dragonFLY, reported in abstract form only $[15,16]$. Two of these cases died prior to presentation to the ED and, therefore, there is no information on symptoms associated with ingestion $[15,16]$. In the other 2 cases, use of Bromo-dragonFLY was associated with significant peripheral vasoconstriction and limb ischemia [15]. However, all of these cases have only been presented in abstract form, with no Bromo-dragonFLY concentrations, and only limited information included on the extent of toxicological screening undertaken to exclude other potential causes for the symptoms and signs. Our case confirmed the presence of Bromo-dragonFLY, ketamine, and cannabis taken by the patient; the delayed onset of symptoms described here is in keeping with what has been previously reported by users of Bromo-dragonFLY.

We have already reported that proactive ad hoc toxicological screening undertaken in recreational drug presentations, in which individuals self-report ingestion of novel drugs or have atypical presentations following ingestion of classical recreational drugs, can detect a range of novel or emerging recreational drugs [1-5]. This case also highlights that research chemical compounds, such as Bromo-dragonFLY, may also enter the recreational drug market. It provides yet further support for the idea that a more systematically-funded approach to toxicological screening in patients with recreational drug toxicity is urgently required. Although this is unlikely to affect the management of an individual patient, as often the results are not available in real time, it would allow clinical toxicologists to monitor changing trends of recreational drug use and provide evidence for legislative authorities to assist them in revising the frameworks under which recreational drugs are currently controlled. 


\section{REFERENCES}

1. Wood DM, Button J, Lidder S, et al. Dissociative and sympathomimetic toxicity associated with recreational use of 1-(3-trifluoromethylphenyl) piperazine (TFMPP) and 1-Benzylpiperazine (BZP). J Med Toxicol. 2008;4:254-257.

2. Dargan PI, Button J, Hawkins L, et al. Detection of the pharmaceutical agent glaucine as a recreational drug. Eur J Clin Pharmacol. 2008;64:553-554.

3. Ovaska H, Viljoen A, Puchnarewicz M, et al. First case report of recreational use of 2,5-dimethoxy-4-chloroamphetamine (DOC) confirmed by toxicological screening. Eur J Emerg Med. 2008;15:354-356.

4. Lidder S, Dargan PI, Sexton M, et al. Cardiovascular toxicity associated with recreational use of diphenylprolinol (diphenyl-2pyrrolidinemethanol (D2PM)). J Med Toxicol. 2008;4:167-169.

5. Wood DM, Dargan PI, Button J, et al. Collapse, reported seizure—and an unexpected pill. Lancet. 2007;369:1490.

6. Balíková M. Nonfatal and fatal DOB (2,5-dimethoxy-4bromoamphetamine) overdose. Forensic Sci Int. 2005;153:85-91.

7. Parker MA, Marona-Lewicka D, Lucaites VL, et al. A novel (benzodifuranyl)aminoalkane with extremely potent activity at the 5-HT2A receptor. J Med Chem. 1998;41:5148-5149.

8. Parker MA, Marona-Lewicka D, Kurrasch D, et al. Synthesis and pharmacological evaluation of ring-methylated derivatives of 3,4-(methylenedioxy)amphetamine (MDA). J Med Chem. 1998;41:1001-1005.
9. Chambers JJ, Kurrasch-Orbaugh DM, Parker MA, et al. Enantiospecific synthesis and pharmacological evaluation of a series of super-potent, conformationally restricted 5-HT(2A/2C) receptor agonists. J Med Chem. 2001;44:1003-1010.

10. Chambers JJ, Parrish JC, Jensen NH, et al. Synthesis and pharmacological characterization of a series of geometrically constrained 5-HT(2A/2C) receptor ligands. J Med Chem. 2003;46: 3526-3535.

11. Drugs-Forum research chemicals discussion forum [webpage on the Internet]. Bromo-Dragonfly (Br-DFLY) trip reports [updated 2007 May 23; cited 2009 Jan 22]. Available from: http://www.drugs-forum.co.uk/forum/showthread.php? $\mathrm{t}=41032$

12. Drugs-Forum research chemicals discussion forum [webpage on the Internet]. Bromo-dragonfly [updated 2008 May 30; cited 2009 Jan 22]. Available from: http://www.drugs-forum.com/ forum/showthread.php?t=30050\&highlight=bromodragonfly

13. Ricaurte GA, McCann UD. Recognition and management of complications of new recreational drug use. Lancet. 2005;365: 2137-2145.

14. Pomarol-Clotet E, Honey GD, Murray GK, et al. Psychological effects of ketamine in healthy volunteers. Phenomenological study. Br J Psychiatry. 2006;189:173-179.

15. Peronne M, Hultén P. Bromo-dragonfly, a life-threatening designer drug. Clin Toxicol. 2008;46:379-380.

16. Andreasen MF, Telving R, Birkler R, et al. A fatal poisoning involving Bromo-Dragonfly. Ann Toxicol Anal. 2008;20(S1):1-55. 